\title{
Equipamentos de proteção individual para prevenção de infecção por SARS-COV-2
}

\author{
Individual protection equipment for the prevention of SARS-COV-2 infection \\ Equipo de protección individual para la prevención \\ de infeccion por SARS-COV-2
}
Antonio Rosa de SOUSA NETO(1)
Bruno Batista BORTOLUZZI (2)
Daniela Reis Joaquim de FREITAS ${ }^{(3)}$

Recebido: 18 abr 2020

Revisado: 25 abr 2020

Aceito: 28 abr 2020

\section{Autor de}

correspondência:

Antonio Rosa de Sousa

Neto

antonioneto@ufpi.edu.br

Conflito de interesses: Os autores declaram não haver nenhum interesse profissional ou pessoal que possa gerar conflito de interesses em relação a este manuscrito.
(1) Universidade Federal do Piauí - UFPI, Departamento
${ }^{(2)}$ Centro Universitário UniCesumar, Curitiba, PR, Brasil.

${ }^{(3)}$ Universidade Federal do Piauí - UFPI, Departamento de Parasitologia e Microbiologia, Teresina, PI, Brasil.

\section{Resumo}

Frente a pandemia ocasionada por COVID-19, faz-se essencial a adoção de medidas de prevenção e controle de infecção. Realizou-se pesquisa bibliográfica e de diretrizes de sociedades médicas sobre as indicações de uso dos Equipamentos de Proteção Individual por parte dos profissionais que atuam na assistência hospitalar. Existem uma série de equipamentos que devem ser adotados por profissionais de saúde, alguns deles já com recomendações específicas para reutilização, dada sua escassez em função da pandemia. Faz-se necessário que os profissionais de saúde se mantenham atualizados e trabalhem conforme as recomendações nacionais e mundiais de saúde.

Descritores: Infecções por Coronavirus; Equipamento de Proteção Individual; Controle de Infecções.

\section{Abstract}

In the face of the pandemic caused by COVID-19, it is essential to adopt infection prevention and control measures. Bibliographical research and guidelines from medical societies were carried out on the indications for the use of Personal Protective Equipment by professionals working in hospital care. There are a series of equipment that must be adopted by health professionals, some of them with specific recommendations for reuse, given their scarcity due to the pandemic. It is necessary for health professionals to keep up to date and work in accordance with national and global health recommendations.

Keywords: Coronavirus Infections; Personal Protective Equipment; Infection Control.

\section{Resumen}

Ante la pandemia causada por COVID-19, es esencial adoptar medidas de prevención y control de infecciones. La investigación bibliográfica y las pautas de las sociedades médicas se llevaron a cabo sobre las indicaciones para el uso de equipos de protección personal por parte de profesionales que trabajan en la atención hospitalaria. Hay una serie de equipos que deben ser adoptados por profesionales de la salud, algunos de ellos con recomendaciones específicas para su reutilización, dada su escasez debido a la pandemia. Es necesario que los profesionales de la salud se mantengan actualizados y trabajen de acuerdo con las recomendaciones sanitarias nacionales y mundiales.

Palabras-claves: Infecciones por Coronavirus; Equipo de Protección Personal; Control de Infecciones. 


\section{Introdução}

Frente a atual problemática que é a pandemia ocasionada pelo vírus denominado SARS-CoV-2 (Síndrome Respiratória Aguda Grave-Coronavírus-2), causador da COVID-19 (Corona Virus Disease 2019), e do grande potencial de transmissão do vírus e disseminação (podendo ocorrer através de gotículas ou contato), assim como a possibilidade elevada de acometer profissionais de saúde que atuam diretamente na assistência prestada aos pacientes contaminados, faz-se essencial a adoção de medidas de prevenção de infecção a fim de diminuir as contaminações secundárias. ${ }^{1}$ Assim, este artigo tem como objetivo discutir sobre as indicações relacionadas ao uso dos Equipamentos de Proteção Individual EPI por parte dos profissionais que atuam na assistência prestada aos pacientes contaminados ou suspeitos de coronavírus, a fim de reduzir ou impedir o aumento dos casos nestes profissionais.

\section{Desenvolvimento}

O primeiro EPI a ser discutido são as máscaras de proteção respiratória (N95, N99, N100, PFF2 ou PFF3), que não possuem evidências de maior proteção relacionada a prevenção de infecções respiratórias virais quando comparadas com as máscaras médicas. No entanto, neste momento de pandemia, a sua indicação de uso é exclusiva para os profissionais de saúde que estão atuando na assistência aos pacientes e realizando procedimentos que geram aerossóis. Seu uso tem sido recomendado juntamente com a utilização de gorros, ${ }^{2}$ para a maior proteção do profissional. Devido à atual situação de escassez de EPI em todo o mundo por conta da pandemia, o Ministério da Saúde vem aconselhando que nos hospitais do Brasil as máscaras sejam reaproveitadas - mas apenas nos casos onde elas estejam íntegras, limpas e com vedação adequada, e a reutilização só é recomendada para o mesmo usuário. ${ }^{3}$

As máscaras cirúrgicas, eficazes quando se quer evitar a transmissão por gotículas respiratórias, são indicadas para todos os profissionais de saúde e equipe de apoio que atuem a uma distância inferior a um metro dos pacientes diagnosticados ou suspeitos por infecção pelo novo coronavírus, assim como para este próprio paciente e seu acompanhante, afim de controlar a fonte de infecção. ${ }^{4}$ Evidencia-se que as mesmas não devem ser reutilizadas nunca, mesmo que passem por processo de limpeza, uma vez que são descartáveis e perdem a efetividade de filtração ao serem umidificadas. Estudos comprovam que a sua utilização por mais de 6 horas, em casos onde não se realiza a troca do EPI, há significativo aumento de risco de auto-contaminação. ${ }^{5}$ 
Referente à utilização de luvas, o uso delas também dependerá do procedimento a ser realizado. Luvas de procedimento não-cirúrgico devem ser utilizadas em qualquer contato com o paciente e superfícies - medida que serve como precaução de contato. Luvas estéreis devem ser utilizadas exclusivamente quando for necessário realização de técnica asséptica, como por exemplo cirurgias; fazendo-se necessário que os profissionais as utilizem de forma adequada e consciente (um par por vez), a fim de evitar que ao invés de proteger, propicie infecção., ${ }^{4,6}$ Os profissionais devem estar atentos também ao hábito de lavagem das mãos - muitas vezes esquecido, antes da realização de procedimentos e após a remoção das luvas, já que as mesmas se configuram como lixo infectante. ${ }^{7}$

Com relação aos óculos de proteção e os protetores faciais (Face Shield-que cobrem tanto a frente como os lados do rosto) - faz-se essencial antes de sua utilização verificar a integridade dos dispositivos, sendo indicado em todos os momentos de prestação de assistência, e principalmente se houver o risco de exposição dos profissionais a secreções corporais - como aquelas advindas da expectoração. É importante que cada profissional possua o seu próprio EPI, realizando a sua limpeza após cada utilização (lavagem com água e sabão ou detergente) e posterior desinfecção com álcool líquido 70\%, ou com outros produtos desinfetantes equivalentes, seguindo o protocolo do hospital ou do fabricante. ${ }^{4,8}$ Os profissionais devem estar atentos quanto aos danos na pele ocorridos em decorrência da utilização por várias horas seguidas de EPIs, servindo como forma de prevenir a irritação e abrasamento da pele o uso de hidratantes ou gel anterior a colocação de óculos e máscaras. $^{9}$

O capote ou avental (gramatura mínima de $30 \mathrm{~g} / \mathrm{m}^{2}$ ) tem como principal função evitar a contaminação da pele e das roupas do profissional que está atuando diretamente com o paciente; referente ao seu uso cabe a cada profissional a avaliação da necessidade de utilização do capote ou avental impermeável (gramatura mínima de $50 \mathrm{~g} / \mathrm{m} 2$ ), conforme o quadro clínico do paciente; a Agência Nacional de Vigilância Sanitária - ANVISA recomenda que o mesmo possua mangas longas, punho de malha ou elástico, abertura posterior, seja confeccionado com material de boa qualidade, proporcione barreira antimicrobiana efetiva, seja confortável e possibilite a movimentação necessária. ${ }^{4}$

A ordem de colocação e retirada dos EPIs conforme o Centro de Controle e Prevenção de Doenças (CDC - Center for Disease Control and Prevention) ${ }^{10}$ está simplificada na Figura 1. Vale ressaltar que pode haver mais de um método aceitável, tratando-se o descrito neste artigo apenas como uma das formas que podem ser seguidas. 


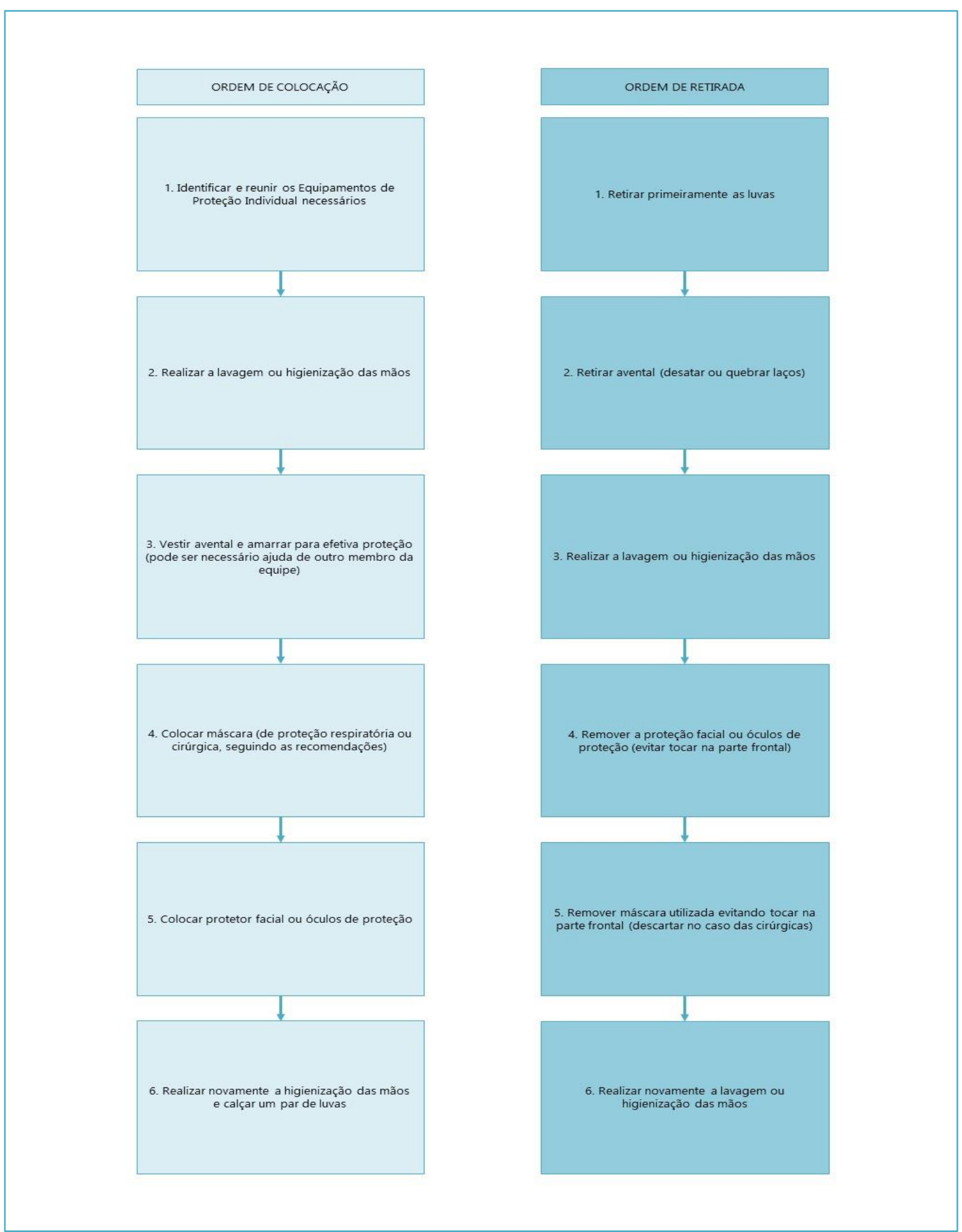

Figura 1. Ordem de colocação e retirada de Equipamentos de Proteção Individual, baseada nas recomendações dos Centros de Controle e Prevenção de Doenças - CDC. Fonte: CDC (2020). ${ }^{10}$ 


\section{Considerações finais}

Resumindo, cada tipo de equipamento de proteção individual possui a sua indicação de uso, mas é fato que diante da atual problemática ocasionada pela pandemia de COVID19 faz-se necessário que os profissionais de saúde se mantenham protegidos, atualizados e trabalhem em conformidade com as recomendações nacionais e mundiais de saúde, não esquecendo de seguir também as outras formas de prevenção.

\section{Referências}

1. Lai $C$, Shih $T$, Ko W, Tang H, Hsueh P. Severe acute respiratory syndrome coronavirus 2 (SARS-CoV-2) and coronavirus disease-2019 (COVID-19): the epidemic and the challenges. Int J Antimicrob Agents. 2020;55(3):105924. https://doi.org/10.1016/j.ijantimicag.2020.105924.

2. Bein B, Bachmann $M$, Huggett $S$, Wegermann P. [SARS CoV-2/COVID-19: EvidenceBased Recommendation on Diagnosis and Therapy]. Anasthesiol Intensivmed Notfallmed Schmerzther. 2020;55(4):257-65. https://doi.org/10.1055/a-1146-8674.

3. Agência Nacional de Vigilância Sanitária. Orientações gerais: racionalização do uso de respiradores descartáveis nos estabelecimentos fabricantes de medicamentos, insumos farmacêuticos, produtos para saúde, cosméticos e saneantes. Brasília, DF: ANVISA; 2020. Disponível em:

http://portal.anvisa.gov.br/documents/219201/4340788/Nota +t\%C3\%A9cnica+respirad ores/c14b21ab-fab7-498b-bfe3-e576ed2b1e67.

4. Agência Nacional de Vigilância Sanitária. Nota técnica GVIMS/GGTES/ANVISA n. 04/2020: orientações para serviços de saúde: medidas de prevenção e controle que devem ser adotadas durante a assistência aos casos suspeitos ou confirmados de infecção pelo novo coronavírus (SARS-CoV-2). Brasília, DF: ANVISA; 2020. Disponível em: http://portal.anvisa.gov.br/documents/33852/271858/Nota+T\%C3\%A9cnica + n+042020+GVIMS-GGTES-ANVISA-ATUALIZADA/ab598660-3de4-4f14-8e6f-b9341c196b28.

5. Chughtai AA, Stelzer-Braid S, Rawlinson W, Pontivivo G, Wang Q, Pan Y, et al. Contamination by respiratory viruses on outer surface of medical masks used by hospital healthcare workers. BMC Infect Dis. 2019 Jun 3;19(1):491. https://doi.org/10.1186/s12879-019-4109-x. 
6. Wilson J, Bak A, Whitfield A, Dunnett A, Loveday H. Public perceptions of the use of gloves by healthcare workers and comparison with perceptions of student nurses. J Infect Prev. 2017;18(3):123-32. https://doi.org/10.1177/1757177416680442.

7. Chang NN, Reisinger HS, Schweizer ML, Jones M, Chrischilles E, Chorazy M, et al. Hand hygiene compliance at critical points of care. Clin Infect Dis. 2020 Feb 8:ciaa130. https://doi.org/10.1093/cid/ciaa130.

8. World Health Organization. Rational use of personal protective equipment (PPE) for coronavirus disease (COVID-19): interim guidance, 19 March 2020. Geneva: WHO; 2020. Disponível em: https://apps.who.int/iris/handle/10665/331498.

9. Yan Y, Chen H, Chen L, Cheng B, Diao P, Dong L, et al. Consensus of Chinese experts on protection of skin and mucous membrane barrier for health-care workers fighting against coronavirus disease 2019. Dermatol Ther. 2020 Mar 13;e13310. https://doi.org/10.1111/dth.13310.

10. Centers for Disease Control and Prevention. Using Personal Protective Equipment (PPE). Atlanta: CDC; 2020. Disponível em: https://www.cdc.gov/coronavirus/2019ncov/hcp/using-ppe.html. 
ISSN 2179-6750

\section{Minicurrículo}

Antonio Rosa de Sousa Neto | ORCiD: 0000-0002-0675-0916

Graduando em Enfermagem pela Universidade Federal do Piauí (UFPI). Membro do Núcleo de Estudos em Microbiologia e Parasitologia (NUEMP) e do Núcleo de Pesquisas em Prevenção e Controle de Infecção em Serviços de Saúde (NUPCISS). Atuando principalmente na área de Prevenção e Controle de Infecções em Serviços de Saúde.

Bruno Batista Bortoluzzi | ORCiD: 0000-0002-0012-3508

Bacharel em Ciências Biológicas pela Universidade do Oeste de Santa Catarina (UNOESC). Mestre em Microbiologia, Parasitologia e Patologia pela Universidade Federal do Paraná (UFPR) e licenciando em Ciências Biológicas pelo Centro Universitário Leonardo da Vinci (UNIASSELVI). Possui experiência em análises clínicas. Professor Universitário no Centro Universitário UniCesumar - Curitiba, lecionando para os cursos de Ciências Biológicas, Enfermagem, Nutrição e Medicina Veterinária.

Daniela Reis Joaquim de Freitas | ORCiD: 0000-0002-5632-0332

Graduada em Ciências Biológicas pela Universidade Federal do Rio Grande do Sul (UFRGS). Mestre em Biologia Celular e Molecular pela Universidade Federal do Rio Grande do Sul (UFRGS). Doutora em Ciências pela Universidade Federal do Rio Grande do Sul (UFRGS). Pós Doutora na área de Ciências Médicas pela Fundação Universidade Federal de Ciências da Saúde de Porto Alegre (UFCSPA). Professora Adjunta III do Departamento de Parasitologia e Microbiologia da Universidade Federal do Piauí (UFPI). Líder do Núcleo de Estudos em Microbiologia e Parasitologia (NUEMP). Docente do Programa de Pós-Graduação em Enfermagem (PPGEnf-UFPI). 